\title{
Some Nonnegative Trigonometric Polynomials Connected With a Problem in Probability
}

\author{
Eugene Lukacs ${ }^{1}$ and Otto Szász ${ }^{2}$
}

\begin{abstract}
Let $0<b_{1}<b_{2}<\ldots<b_{n}$ be integers and let $g(\theta)$ be the Vandermonde determinant formed from $b_{1}^{2}, b_{2}^{2}, \ldots, b_{n}^{2}$ with the first row replaced by $\sin ^{2}\left(b_{i} \theta / 2\right)(i=1, \ldots, n)$. The function $g(\theta)$ is then a cosine polynomial. In connection with a problem in probability, the question arose as to when $g(\theta)$ is a nonnegative trigonometric polynomial. This question is answered for six classes of such trigonometric polynomials.
\end{abstract}

In a previous paper ${ }^{3}$ we gave a necessary condition that a polynomial without multiple roots must satisfy in order that its reciprocal be the Fourier transform of a distribution function. Imposing a further restriction on the polynomials it is easy to derive the following condition:

The reciprocal of a polynomial whose roots are all single and have the same imaginary part is the Fourier transform of a distribution function if and only if

(1) The polynomial has one purely imaginary root ai $(a \neq 0)$ and $n$ pairs of complex roots $\pm b_{k}+a i$ $\left(0<b_{1}<b_{2}<\ldots .<b_{n}, k=1,2, \ldots, n\right)$.

(2) The determinant

$\left|\begin{array}{ccc}\sin ^{2} \frac{b_{1} \theta}{2}, & \sin ^{2} \frac{b_{2} \theta}{2}, \ldots, & \sin ^{2} \frac{b_{n} \theta}{2} \\ b_{1}^{2}, & b_{2}^{2}, \ldots, & b_{n}^{2} \\ \cdot & \ldots & \cdot \\ \cdot & \cdot & \cdot \\ b_{1}^{2(n-1)}, & b_{2}^{2(n-1)}, \ldots, & b_{n}^{2(n-1)}\end{array}\right| \geq 0$ for all $\theta$.

This condition follows easily from formula (4.4) of the reference cited in footnote 3 . It is therefore of some interest to study this determinant and to investigate for what values of $b_{1}, b_{2}, \ldots, b_{n}$ it is a nonnegative function of $\theta$.

In this paper we consider this determinant only for integer values of the $b_{i}$ and show that it represents for certain configurations of the $b_{i}$ a nonnegative trigonometric polynomial. Certain relations for generalized Vandermonde determinants of odd integers are also obtained.

We introduce first some notations. Let $0<b_{1}<b_{2}<\ldots<b_{n}$ be $n$ integers and

$$
V_{k}=\left|\begin{array}{ccc}
b_{1}^{2 k}, & b_{2}^{2 k}, \ldots, & b_{n}^{2 k} \\
b_{1}^{2}, & b_{2}^{2}, \ldots, & b_{n}^{2} \\
\cdot & \cdot \ldots & \cdot \\
\cdot & \cdot & \cdot \\
b_{1}^{2(n-1)}, & b_{2}^{2(n-1)}, \ldots, & b_{n}^{2(n-1)}
\end{array}\right|
$$

1 National Bureau of Standards and Our Lady of Cincinnati College,

2 University of Cincinnati and National Bureau of Standards. dian J. Math. 3, 140 to 144 (1951). the generalized Vandermonde determinant formed from the numbers $b_{1}^{2}, b_{2}^{2}, \ldots, b_{n}^{2}$ with the exponents $k, 1,2, \ldots,(n-1)$. Clearly $V_{1}=V_{2}=\ldots=$ $V_{n-1}=0$, while $V_{0}$ is the ordinary Vandermonde determinant of the numbers $b_{1}^{2}, \ldots, b_{n}^{2}$. Denote further by $\Delta_{m}$ the minor of the element in the first row and $m$-th column of $V_{0}$ so that $T_{0}=\sum_{m=1}^{n}(-1)^{m-1} \Delta_{m}$. In this paper we study the function

$$
\begin{aligned}
& \sin ^{2} \frac{b_{1} \theta}{2}, \quad \sin ^{2} \frac{b_{2} \theta}{2}, \ldots, \quad \sin ^{2} \frac{b_{n} \theta}{2} \\
& b_{1}^{2}, \quad b_{2}^{2}, \ldots, \quad b_{n}^{2} \\
& g(\theta)=2 \\
& b_{1}^{2(n-1)} \\
& b_{2}^{2(n-1)}, \ldots \\
& =V_{0}+\sum_{m=1}^{n}(-1)^{m} \Delta_{m} \cos b_{m} \theta
\end{aligned}
$$

For the discussion of $g(\theta)$ we need the following lemmas:

Lemma 1. If $b_{1}, b_{2}, \ldots, b_{n}$ are integers, the determinant (2) can be factored so that

$$
g(\theta)=(1-\cos \theta)^{n} A(\cos \theta),
$$

where $A(x)$ is a polynomial in $x$ of degree $b_{n}-n$.

Lemma 2. If $b_{1}, b_{2}, \ldots, b_{n}$ are odd integers we have

$g^{\prime}(\theta)=\sin \theta\left(1-\cos ^{2} \theta\right)^{n-1} B(\cos \theta)=(\sin \theta)^{2 n-1} B(\cos \theta)$,

where $B(x)$ is a polynomial in $x$ of degree $b_{n}-2 n+1$.

To prove lemma 1 we differentiate (2) with respect to $\theta$ and then set $\theta=0$. This shows that
$g^{2 j-1}(0)=0$
for $j=1,2, \ldots$ ad inf.
$g^{2 j}(0)=0$
for $j=0,1,2, \ldots(n-1)$
$g^{2 k}(0)=(-1)^{k-1} V_{k}$
for $k=n,(n+1), \ldots$ ad inf. 
From (5) we obtain the expansion of $g(\theta)$ into a series

$$
g(\theta)=\sum_{k=n}^{\infty}(-1)^{k-1} \frac{V_{k}}{(2 k) !} \theta^{2 k}
$$

On the other hand, (2) indicates that $g(\theta)$ is a cosine polynomial of degree $b_{n}$ which vanishes for $\theta=0$. Therefore it has the form

$$
\begin{aligned}
g(\theta) & =\beta_{1}(1-\cos \theta)+\beta_{2}(1-\cos \theta)^{2}+\ldots \\
& +\beta_{b_{n}}(1-\cos \theta)^{b_{n}}
\end{aligned}
$$

Comparing (6) and ( 7$)$ it is seen that $\beta_{1}=\beta_{2}=\ldots=$ $\beta_{n-1}=0$ so that $(1-\cos \theta)^{n}$ is a factor of $g(\theta)$; this establishes lemma 1.

To prove lemma 2 we assume that all the $b_{i}(i=1$, $2, \ldots, n)$ are odd numbers. It follows then from (2) that

therefore

$$
g(\pi-\theta)=2 V_{0}-g(\theta),
$$

From (3) we have

$$
g^{\prime}(\pi-\theta)=g^{\prime}(\theta)
$$

$$
\begin{aligned}
g^{\prime}(\theta)= & (1-\cos \theta)^{n-1} \sin \theta[n A(\cos \theta)- \\
& \left.(1-\cos \theta) A^{\prime}(\cos \theta)\right] .
\end{aligned}
$$

Substituting this into (9) we obtain

$$
\begin{aligned}
& (1-\cos \theta)^{n-1} \sin \theta\left[n A(\cos \theta)-(1-\cos \theta) A^{\prime}(\cos \theta)\right] \\
= & (1+\cos \theta)^{n-1} \sin \theta[n A(-\cos \theta)- \\
& \left.(1+\cos \theta) A^{\prime}(-\cos \theta)\right] .
\end{aligned}
$$

This shows that $n A(\cos \theta)-(1-\cos \theta) A^{\prime}(\cos \theta)$ and therefore also $g^{\prime}(\theta)$ has the factor $(1+\cos \theta)^{n-1}$. This completes the proof of lemma 2.

If we introduce as a new variable

$$
x=\cos \theta
$$

and write

$$
P(x)=g(\arccos x)
$$

we have from lemma 1

$$
P(x)=(1-x)^{n} A(x),
$$

and similarly from lemma 2

$$
P^{\prime}(x)=-\left(1-x^{2}\right)^{n-1} B(x) .
$$

We substitute next the expansion (6) into (8) and see that

$$
2 V_{0}-\sum_{k=n}^{\infty}(-1)^{k-1} \frac{V_{k}}{(2 k) !} \theta^{2 k}=\sum_{k=n}^{\infty}(-1)^{k-1} \frac{V_{k}}{(2 k) !}(\pi-\theta)^{2 k}
$$

Differentiating (14) and setting $\theta=0$ we obtain from this equation a number of relations for generalized Vandermonde determinants formed of odd integers

$$
\left.\begin{array}{c}
2 V_{0}=\sum_{k=n}^{\infty}(-1)^{k-1} \frac{V_{k}}{(2 k) !} \pi^{2 k} \\
\begin{array}{c}
\sum_{k=n}^{\infty}(-1)^{k-1} \frac{V_{k}}{(2 k-m) !} \pi^{2 k}=0 \\
\text { for } m=1,2, \ldots(2 n-1)
\end{array} \\
\begin{array}{r}
\sum_{k=n+m}^{\infty}(-1)^{k-1} \frac{V_{k} \pi^{2 k}}{(2 k-2 n-2 m) !} \\
\quad=(-1)^{n+m} \pi^{2(n+m)} V_{n+m} \\
\text { for } m=0,1,2, \ldots \text { ad inf. } \\
\sum_{k=n+m+1}^{\infty}(-1)^{k-1} \frac{V_{k} \pi^{2 k}}{(2 k-2 n-2 m-1) !}=0 \\
\text { for } m=0,1,2, \ldots \text { ad inf. }
\end{array}
\end{array}\right\}
$$

In the following we discuss several configurations of the integers $b_{1}, b_{2}, \ldots, b_{n}$, which lead to nonnegative trigonometric polynomials $g(\theta)$, the results are given in statements, labelled (A) (B) ... (F). (A) If the $b_{i}$ are the first $n$ consecutive integers, that is if $b_{i}=i$ for $i=1,2, \ldots, n$, then the trigonometric polynomial $g(\theta)$ is nonnegative.

Proof: From lemma 1 we see $g(\theta)=A(1-\cos \theta)^{n}$, where $A$ is a constant. Hence $A=2^{-n} g(\pi)$; from (2) it is seen that $g(\pi)=V_{0}+\sum_{m=1}^{n} \Delta_{m}$. Therefore $A>0$ and consequently $g(\theta)$ nonnegative for all values of $\theta$. (B) If the $b_{i}$ are the first $n$ consecutive odd integers, that is, if $b_{i}=2 i-1$ for $i=1,2, \ldots, n$ then the trigonometric polynomial $g(\theta)$ is nonnegative.

Proof: Since $b_{n}=2 n-1$ the polynomial $B(x)$ of lemma 2 reduces to a constant $B$. From (13) we have $P^{\prime}(x)=-B\left(1-x^{2}\right)^{n-1}$ or, in view of $P(1)=0$, $P(x)=B \int_{x}^{1}\left(1-t^{2}\right)^{n-1} d t$.

Formulae (11) and (2) show then that $P(0)=g(\pi / 2)=$ $V_{0}>0$, hence

$$
B=\frac{P(0)}{\int_{0}^{1}\left(1-t^{2}\right)^{n-1} d t}>0 .
$$

Therefore $P(x) \geq 0$ for $|x| \leq 1$. This completes the proof of statement (B) since the inequalities $P(x) \geq 0$ for $|x| \leq 1$ and $g(\theta) \geq 0$ for all $\theta$ are equivalent. (C) If $b_{n}=n+1$, that is if the numbers $b_{1}, \ldots, b_{n}$ are obtained from the first consecutive $(n+1)$ integers by omitting the integer $k(1 \leq k \leq n)$ then the trigonometric polynomial $g(\theta)$ is nonnegative if and only if $2 k^{2} \geq(n+1)$.

Proof: In this case $P(x)$ has degree $(n+1)$ so that according to lemma $1 A(x)$ is a linear function.

$$
A(x)=a+b x .
$$


The substitution (10) transforms $\cos k \theta$ into $T_{k}(x)$ where $T_{k}(x)$ is the $k$-th Tchebicheff polynomial of the first kind. Formulae (2), (3) and (16) show that

$P(x)=(1-x)^{n}(a+b x)=V_{0}+\sum_{m=1}^{n}(-1)^{m} \Delta_{m} T_{b_{m}}(x)$.

The coefficients $a$ and $b$ of $A(x)$ can be determined from (17), after some elementary computations it is seen that

$$
A(x)=2^{n} \Delta_{n}\left[x+\frac{n(n+1)+k^{2}}{(n+1)^{2}-k^{2}}\right] .
$$

In the interval $|x|<1$ the functions $A(x)$ and $P(x)=$ $(1-x)^{n} A(x)$ have the same sign so that it is sufficient to determine when $A(x)$ is nonnegative in $|x|<1$. From (18) it is seen that $A(x) \geq 0$ in $|x|<1$ if and only if $n(n+1)+k^{2} \geq(n+1)^{2}-k^{2}$, that is if $2 k^{2} \geq n+1$.

(D) If the $b_{i}(i=1,2, \ldots, n)$ are all odd numbers and if $b_{n}=2 n+1$ so that $b_{1}, b_{2}, \ldots, b_{n}$ are obtained from the first $(n+1)$ odd integers by omitting the $k$-th odd integer $2 k-1(1 \leq k \leq n)$, than the trigonometric polynomial $g(\theta)$ is nonnegative if either one or the other of the following conditions is satisfied

$$
\begin{aligned}
& k \geq \frac{1}{2}(1+\sqrt{1+2 n}) \\
& k<\frac{1}{2}(1+\sqrt{1+2 n}),
\end{aligned}
$$

but

$$
\frac{n-2 k(k-1)}{2[n(n+1)-k(k-1)]} \leq z_{n}^{2}
$$

where $z_{n}$ is the root of the equation

$$
\int_{-z}^{1}\left(1-t^{2}\right)^{n-1}\left(t^{2}-z^{2}\right) d t=0
$$

which falls into the interval $(0,1)$.

If neither (i) nor (ii) is satisfied, then the function $g(\theta)$ assumes also negative values. Moreover it is possible to simplify (ii) for large $n$ by proving the Corollary to statement $(D)$.

If for large $n$ and $k<[1+\sqrt{1+2 n}] / 2$ also

$$
\sqrt{\frac{\left(n+\frac{1}{2}\right)[n-2 k(k-1)]}{2[n(n+1)-k(k-1)]}} \leq \rho=0.5939157 \ldots
$$

then $g(\theta)$ is nonnegative; if on the other hand

$$
\frac{\left(n+\frac{1}{2}\right)[n-2 k(k-1)]}{2[n(n+1)-k(k-1)]}>\rho^{2}
$$

then there are integers $n$ and $k$ for which $g(\theta)$ assumes positive and negative values.
To prove statement (D) we need the following lemma

Lemma 3. Let

$$
P_{s}(x)=\int_{x}^{1}\left(1-t^{2}\right)^{n-1} t^{2 s} d t \text { for } s=0,1
$$

and

$$
Q(x, \zeta)=P_{1}(x)-\zeta^{2} P_{0}(x)
$$

The polynomial $Q(x, \zeta)$ is nonnegative in the interval $-1 \leq x \leq 1$ if and only if

$$
\zeta \leq z_{n}
$$

Here $z_{n}$ is the root of the equation

$$
R(z) \equiv Q(-z, z) \equiv \int_{-z}^{1}\left(1-t^{2}\right)^{n-1}\left(t^{2}-z^{2}\right) d t=0,
$$

which falls into the interval $(0,1)$.

$$
\text { Moreover let } w_{n}=\sqrt{n+\frac{1}{2}} z_{n} \text {. }
$$

Then $\lim _{n \rightarrow \infty} w_{n}=\rho$ exists and is the root of the equation

$$
F(\rho)=\sqrt{\pi}\left(\frac{1}{2}-\rho^{2}\right)-2 \rho^{3} \int_{0}^{1}\left(1-t^{2}\right) e^{-\rho^{2} t^{2}} d t=0,
$$

which is located in the interval $\left(0, \sqrt{\frac{1}{2}}\right)$.

Some values of $z_{n}$ as well as $\rho$ were computed. The proof of this lemma is rather lengthy. In order to avoid interrupting the discussion of the various nonnegative trigonometric polynomials, this proof will be given in the last section of this paper.

We proceed now to the proof of statement (D).

According to lemma $2 B(x)$ is a polynomial of the second degree. Since the $b_{i}$ are all odd numbers $P^{\prime}(x)$ and $B(x)$ are even functions of $x$ so that one obtains from (13) and (2)

$$
P^{\prime}(x)=-\left(1-x^{2}\right)^{n-1}\left(a+c x^{2}\right)=\sum_{m=1}^{n}(-1)^{m} \Delta_{m} T_{b_{m}}^{\prime}(x) .
$$

This relation permits us to determine $a$ and $c$, and it is seen that

$P^{\prime}(x)=$
$-2^{2 n}(2 n+1) \Delta_{n}\left(1-x^{2}\right)^{n-1}\left\{x^{2}-\frac{n-2 k(k-1)}{2[n(n+1)-k(k-1)]}\right\}$,

If we use for brevity the notation

and

$$
\gamma=2^{2 n}(2 n+1) \Delta_{n}
$$

$$
\zeta_{n, k}^{2}=\frac{n-2 k(k-1)}{2[n(n+1)-k(k-1)]},
$$


we have

$$
\left.\begin{array}{l}
P^{\prime}(x)=-\gamma\left(1-x^{2}\right)^{n-1}\left(x^{2}-\zeta_{n, k}^{2}\right) \\
P(x)=\gamma \int_{x}^{1}\left(1-t^{2}\right)^{n-1}\left(t^{2}-\zeta_{n, k}^{2}\right) d t
\end{array}\right\}
$$

If (i) is satisfied we see that $\zeta_{n, k}^{2} \leq 0$, so that from $(27)$ $P^{\prime}(x) \leq 0$ for $|x| \leq 1$. In this case $P(x)$ is nonincreasing in the interval $-1 \leq x \leq+1$, since $P(1)=0$ this means $P(x) \geq 0$ for $|x| \leq 1$ so that also $g(\theta) \geq 0$.

We assume next that (ii) is satisfied, then $\zeta_{n, k}^{2}>0$. Since $\gamma>0$ and $P(x)=\gamma Q\left(x, \zeta_{n, k}\right)$, we see that statement (D) follows immediately from lemma 3.

(E) If the numbers $b_{1}, \ldots, b_{n}$ are obtained from the first consecutive $(n+2)$ integers by omitting the two integers $k$ and $p$ where $1 \leq k \leq p-1 \leq n$, then $g(\theta)$ is nonnegative if and only if a certain polynomial $Q(x)$ of the second degree is nonnegative for $|x| \leq 1$. Here

$$
\begin{aligned}
Q(x)= & 4 x^{2}+4[n-L(n, k, p)] x+2 n^{2}+(n-2)- \\
& 4 n L(n, k, p)+N(n, k, p),
\end{aligned}
$$

where

$$
\left.\begin{array}{c}
L(n, k, p)=\frac{(n+2)\left[(n+1)^{2}-k^{2}\right]\left[(n+1)^{2}-p^{2}\right]}{\left[(n+2)^{2}-k^{2}\right]\left[(n+2)^{2}-p^{2}\right]} \\
N(n, k, p)=\frac{(n+2)(2 n+3)\left(n^{2}-k^{2}\right)\left(n^{2}-p^{2}\right)}{\left[(n+2)^{2}-k^{2}\right]\left[(n+2)^{2}-p^{2}\right]}
\end{array}\right\}
$$

Proof: In this case $b_{n}=n+2$, so that according to lemma $1 A(x)$ is a polynomial of the second degree, $A(x)=a x^{2}+b x+c$. From (12), (2) and (3) we have

$$
\begin{aligned}
P(x) & =(1-x)^{n}\left(a x^{2}+b x+c\right) \\
& =V_{0}+\sum_{m=1}^{n}(-1)^{m} \Delta_{m} T_{b_{m}}(x) .
\end{aligned}
$$

From this relation we can determine the coefficients $a, b$, and c. A somewhat tedious elementary computation shows that

$$
A(x)=\left(a x^{2}+b x+c\right)=2^{n-1} \Delta_{n} Q(x) .
$$

This completes the proof of statement (E) which follows immediately from (30).

Certain particular cases can be discussed easily

(a) If $k=p-1=n$ then $N(n, n, n+1)=L(n, n, n+1)$ $=0$ so that $Q(x)=4 x^{2}+4 n x+2 n^{2}+n-2$. The discriminant of $Q(x)$ is then $-16(n-1)(n+2)$ and is negative for $n>1$. Therefore, $Q(x) \geq 0$ for all $x$ so that $\mathrm{g}(\theta)$ must be nonnegative.

(b) If $k<n$ while $p=(n+1)$ then $L(n, k, n+1)=0$ and

$$
N(n, k, n+1)=-\frac{(n+2)(2 n+1)\left(n^{2}-k^{2}\right)}{(n+2)^{2}-k^{2}} .
$$

$Q(x)$ and its discriminant can be computed easily and it can be shown that $g(\theta)$ is nonnegative if and only if

$$
k^{2}>\frac{(n-1)(n+2)}{2 n} .
$$

(c) If $k=n-1$ and $\mathrm{p}=n$ then $N(n, n-1, n)=0$ and

$$
L(n, n-1, n)=\frac{n(n+2)}{3(n+1)} .
$$

The discriminant of $Q(x)$ is then

$$
\frac{16}{9(n+1)^{2}}\left(-2 n^{4}-5 n^{3}+7 n^{2}+27 n+18\right),
$$

which is negative if $n \geq 3$ so that in this case $g(\theta) \geq 0$. If $n=2, Q(x)$ has two roots in the interval $-1 \leq x \leq+1$ so that $g(\theta)$ assumes also negative values

We proceed to discuss a more complicated case by assuming that the $b_{1}, b_{2}, \ldots, b_{n}$ are obtained from the first $(n+2)$ odd integers by omitting two odd integers $(2 k-1)$ and $(2 p-1)$ where

$$
1 \leq k \leq p-1 \leq n
$$

so that $b_{n}=2 n+3$.

We see then from lemma 2 that $B(x)$ is a polynomial of degree four. By the procedure employed in case $(\mathrm{C})$ it is possible to determine the coefficients of this polynomial.

We obtain

$$
P^{\prime}(x)=-\gamma\left(1-x^{2}\right)^{n-1} B(x)=-\gamma\left(1-x^{2}\right)^{n-1}\left(4 x^{4}+a x^{2}-b\right)
$$

Here

$$
\left.\begin{array}{l}
\gamma=2^{2 n}(2 n+3) \Delta_{n}>0, \\
a=\frac{2\left\{-2(n+1)^{2}(3 n+5)+(n+1)(2 n+5)[k(k-1)+p(p-1)]-4 k(k-1) p(p-1)\right\}}{[(n+2)(n+1)-k(k-1)][(n+2)(n+1)-p(p-1)]} \\
b=\frac{\{-(n+1)(3 n+4)+2(n+1)[k(k-1)+p(p-1)]-4 k(k-1) p(p-1)\}}{[(n+2)(n+1)-k(k-1)][(n+2)(n+1)-p(p-1)]}
\end{array}\right\} .
$$


The trigonometric polynomial $g(\theta)$ is nonnegative if and only if

$P(x)=\gamma \int_{x}^{1}\left(1-t^{2}\right)^{n-1}\left(4 t^{4}+a t^{2}-b\right) d t \geq 0$ for all $|x| \leq 1$.

The nonnegativity of the polynomial $P(x)$ depends on the nature of the roots of the polynomial $B(x)=4 x^{4}+a x^{2}-b$. This introduces the need to distinguish a number of cases. For this purpose we write

$$
\left.\begin{array}{l}
h(\tau)=4 \tau^{2}+a \tau-b=4\left(\tau-\tau_{1}\right)\left(\tau-\tau_{2}\right) \text { where } \\
\tau_{1}=\frac{-a-\sqrt{a^{2}+16 b}}{8} \quad \tau_{2}=\frac{-a+\sqrt{a^{2}+16 b}}{8}
\end{array}\right\} .
$$

We are now in a position to formulate statement $(\mathbf{F})$. (F) If the numbers $b_{1}, b_{2}, \ldots, b_{n}$ are obtained from the first consecutive $(n+2)$ odd integers by omitting the two integers $2 k-1$ and $2 p-1$ (where $1 \leq k \leq p-1 \leq n)$ then the trigonometric polynomial $g(\theta)$ is nonnegative if and only if one of the following four, mutually exclusive, conditions is satisfied

( $\alpha) \quad a \geq 0$ and $\mathrm{b} \leq 0$,

( $\beta) \quad a<0$ and $a^{2}+16 b \leq 0$,

( $\gamma) a \geq 0$ and $0<b<4+a$ and also $R_{2}\left(\sqrt{\tau_{2}}\right)-\tau_{1} R_{1}\left(\sqrt{\tau_{2}}\right) \geq 0$.

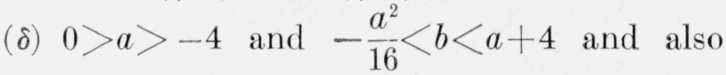
$R_{2}\left(\sqrt{\tau_{2}}\right)-\tau_{1} R_{1}\left(\sqrt{\tau_{2}}\right) \geq 0$, and if $\tau_{1}>0$ $R_{2}\left(-\sqrt{\tau_{1}}\right)-\tau_{2} R_{1}\left(-\sqrt{\tau_{1}}\right) \geq 0$.

Here

$R_{i}(z)=\int_{-z}^{1}\left(1-t^{2}\right)^{n-1} t^{2(i-1)}\left(t^{2}-z^{2}\right) d t$ for $i=1,2$,

so that $R_{1}(z)=R(z)$ as given by $(23)$.

Proof: If $h(\tau)=4 \tau^{2}+a \tau-b \geq 0$ for $0 \leq \tau \leq 1$, then $P^{\prime}(x)=-\gamma\left(1-x^{2}\right)^{n-1} h\left(x^{2}\right) \leq 0$ for $-1 \leq x \leq 1$. Since $P(1)=0$ we see that $\bar{P}(x) \geq 0$ for $-1 \leq x \leq+1$. Clearly $h(\tau)=\left(2 \tau+\frac{a}{4}\right)^{2}-\frac{a^{2}}{16}-b$, therefore

$$
\begin{aligned}
& h(\tau) \geq 0 \text { in } 0 \leq \tau \leq 1 \text { if and only if } \\
& \left(2 \tau+\frac{a}{4}\right)^{2} \geq \frac{a^{2}+16 b}{16} \text { in } 0 \leq \tau \leq 1 .
\end{aligned}
$$

If $a \geq 0$, (38) holds if and only if $b \leq 0$, but this is exactly condition $(\alpha)$.
We next derive the condition for the validity of (37) when $a<0$. Clearly $h^{\prime}(\tau)=8 \tau+a, h^{\prime \prime}(\tau)=8$, so that $h(\tau)$ has a minimum at $\tau=-a / 8$.

We consider first the case $|a| / 8=-a / 8<1$, we have then

$$
\min _{0 \leq \tau \leq 1} h(\tau)=h\left(\frac{-a}{8}\right)=-\frac{a^{2}+16 b}{16} .
$$

Relation (37) is therefore satisfied if $-8<a<0$ and $a^{2}+16 b \leq 0$. If $|a| / 8 \geq 1$ we see that $h^{\prime}(\tau) \leq 8(\tau-1) \leq 0$ in $0 \leq \bar{\tau} \leq 1$, so that $\min _{0 \leq \tau \leq 1} h(\tau)=h(\overline{1})=4+a-b$. The function $h(\tau)$ is nonnegative if $a \leq-8$ and $4+a-b \geq 0$. But $a^{2}+16 b=(a+8)^{2}-16(4+a-b)$ for any $a$, hence if $a^{2}+16 b \leq 0$ we always have $4+a-b>0$. The alternatives discussed for case $a<0$ can therefore be joined so that condition $(\beta)$ is established. To complete the discussion we must consider the remaining cases, namely

$\left(\gamma^{\prime}\right)$

and

$\left(\delta^{\prime}\right)$

$$
a \geq 0 \text { and } b>0 \text {, }
$$

$$
a<0 \text { and } a^{2}+16 b>0 .
$$

In case $\left(\gamma^{\prime}\right)$ we see from (35) that $\tau_{1}<0$ while $\tau_{2}>0$. Since $P(x)=4 \gamma \int_{r}^{1}\left(1-t^{2}\right)^{n-1}\left(t^{2}-\tau_{1}\right)\left(t^{2}-\tau_{2}\right) d t$, it follows that $P(x) \leq 0$ if $\tau_{2} \geq 1$, that is if $\sqrt{a^{2}+16 b} \geq a+8$, but this occurs if and only if $b \geq 4+a$.

Thus $P(x) \leq 0$ for $-1 \leq x \leq 1$ if $a \geq 0$ and $b \geq 4+a$, therefore $\left(\gamma^{\prime}\right)$ reduces to the case

$$
\left(\gamma^{\prime \prime}\right) \quad a \geq 0 \text { and } 0<b<4+a .
$$

In this case both roots $\tau_{1}$ and $\tau_{2}$ are real and a simple computation shows that $\min _{-1<x \leq 1} P(x)=P\left(-\sqrt{\tau_{2}}\right)$, hence $P(x) \geq 0$ in $|x| \leq 1$ if and only if

$$
P\left(-\sqrt{\tau_{2}}\right)=4 \gamma \int_{-\sqrt{\tau_{2}}}^{1}\left(1-t^{2}\right)^{n-1}\left(t^{2}-\tau_{1}\right)\left(t^{2}-\tau_{2}\right) d t \geq 0 .
$$

That is if

$$
\begin{aligned}
& \int_{-\sqrt{\tau_{2}}}^{1}\left(1-t^{2}\right)^{n-1}\left(t^{2}-\tau_{2}\right) t^{2} d t- \\
& \tau_{1} \int_{-\sqrt{\tau_{2}}}^{1}\left(1-t^{2}\right)^{n-1}\left(t^{2}-\tau_{2}\right) d t \geq 0
\end{aligned}
$$

or, written in a more concise notation

$$
R_{2}\left(\sqrt{\tau_{2}}\right)-\tau_{1} R_{1}\left(\sqrt{\tau_{2}}\right) \geq 0,
$$

so that $(\gamma)$ is established.

We proceed with the discussion of the last case $\left(\delta^{\prime}\right) a<0$ and $a^{2}+16 b>0$ and show first that always $a+4>0$. From (33) we obtain easily

$$
\frac{(a+4)}{2}=\frac{2(n+1)^{2}\left(n^{2}+n-1\right)+(n+1)[k(k-1)+p(p-1)]-2 k(k-1) p(p-1)}{[(n+2)(n+1)-k(k-1)][(n+2)(n+1)-p(p-1)]} .
$$


On account of (31) this fraction has always a positive denominator so that its sign is determined by the sign of the numerator which we denote by $M(n, k, p)$. We have then

$$
\begin{aligned}
M(n, k, p)= & p(p-1)[(n+1)-2 k(k-1)]+ \\
& (n+1) k(k-1)+2(n+1)^{2}\left(n^{2}+n-1\right) \\
= & k(k-1)[(n+1)-2 p(p-1)]+ \\
& (n+1) p(p-1)+2(n+1)^{2}\left(n^{2}+n-1\right) .
\end{aligned}
$$

If $(n+1)-2 k(k-1) \geq 0$ then $M(n, k, p)>0$. If on the other hand $(n+1)-2 k(k-1)<0$ then also $(n+1)-2 p(p-1)<0$. Therefore $M(n, k, p)$ decreases for fixed $k$ and increasing $p$ as well as for fixed $p$ and increasing $k$. Hence $M(n, k, p)$ attains its minimum value if $k$ and $p$ are as large as possible, that is

$$
\begin{gathered}
M(n, k, p) \geq M(n, n, n+1) \\
=2(n+1)^{2}\left(n^{2}+n-1\right)-2 n^{2}(n+1)(n-2) \\
\geq 2 n^{2}(n+1)^{2}-2 n^{2}(n+1)(n-2) \text { if } n \geq 1 .
\end{gathered}
$$

Therefore $M(n, k, p) \geq 6 n^{2}(n+1)>0$ if $n \geq 1$ so that also $a+4>0$. It is therefore no restriction if we write the case $\left(\delta^{\prime}\right)$ in the form

$$
0>a>-4 \text { and } a^{2}+16 b>0 \text {. }
$$

We must first consider the case

$$
0>a>-4 \text { and } b \geq a+4 \text {. }
$$

We have then $a^{2}+16 b>0$ so that $h(\tau)$ has two real roots; from (35) we see that $\tau_{1}<0$ while $\tau_{2} \geq 1$. Therefore

$$
P(x)=4 \gamma \int_{x}^{1}\left(1-t^{2}\right)^{n-1}\left(t^{2}-\tau_{1}\right)\left(t^{2}-\tau_{2}\right) d t \leq 0
$$

for $-1 \leq x \leq 1$ so that $\left(\delta_{1}\right)$ does not yield nonnegative trigonometric polynomials. We finally have to investigate the possibility that

$$
0>a>-4 \text { and }-\frac{a^{2}}{16}<b<4+a
$$

holds. Again $a^{2}+16 b>0$ and $h(\tau)$ has two real roots $\tau_{1}$ and $\tau_{2}$. It follows then from (35) that $1 \geq \tau_{2}>\tau_{1}>-1$.

Assume first that $\tau_{1}>0$.

By a simple computation it is seen that $P(x)$ has the two minima $+\sqrt{\tau_{1}}$ and $-\sqrt{\tau_{2}}$ inside the interval $(-1,+1) . \quad P(x)$ is therefore nonnegative for $-1 \leq x \leq 1$ if and only if $P\left(\sqrt{\tau_{1}}\right) \geq 0$ and $P\left(-\sqrt{\tau_{2}}\right) \geq 0$. According to (34) and (35) this means that

$$
\begin{aligned}
\int_{-\sqrt{\tau_{2}}}^{1}\left(1-t^{2}\right)^{n-1} t^{2}\left(t^{2}-\tau_{2}\right) d t- & \\
& \tau_{1} \int_{-\sqrt{\tau_{2}}}^{1}\left(1-t^{2}\right)^{n-1}\left(t^{2}-\tau_{2}\right) d t \geq 0
\end{aligned}
$$

and

$$
\begin{aligned}
& \int_{\sqrt{\tau_{1}}}^{1}\left(1-t^{2}\right)^{n-1} t^{2}\left(t^{2}-\tau_{1}\right) d t- \\
& \tau_{2} \int_{\sqrt{\tau_{1}}}^{1}\left(1-t^{2}\right)^{n-1}\left(t^{2}-\tau_{1}\right) d t \geq 0
\end{aligned}
$$

or, using the notation of (36)

$$
R_{2}\left(\sqrt{\tau_{2}}\right)-\tau_{1} R_{1}\left(\sqrt{\tau_{2}}\right) \geq 0
$$

and simultaneously $R_{2}\left(-\sqrt{\tau_{1}}\right)-\tau_{2} R_{1}\left(-\sqrt{\tau_{1}}\right) \geq 0$.

If $\tau_{1} \leq 0$ then there is only one minimum for $P(x)$, and therefore only the first condition remains. This establishes condition $(\delta)$ and completes the proof of statement $(\mathrm{F})$.

In this section we give proof of lemma 3, which was stated on page 141 . We write

$$
P_{s}(x)=\int_{x}^{1}\left(1-t^{2}\right)^{n-1} t^{2 s} d t \quad(s=0,1)
$$

and want to determine the conditions which $\zeta$ has to satisfy in order that the polynomial

$$
Q(x, \zeta)=P_{1}(x)-\xi^{2} P_{0}(x)
$$

should be nonnegative for $-1 \leq x \leq+1$.

Clearly $0<P_{1}(x)<P_{0}(x)$ for $-1 \leq x<+1$. Therefore $Q(x, \zeta)<0$ for $|x|<1$ if $\zeta \geq 1$. If for some value $\zeta_{0}$ such that $\left|\zeta_{0}\right|<1$ the function $Q\left(x, \zeta_{0}\right) \geq 0$ for $|x| \leq 1$ then also $Q(x, \zeta) \geq 0$ for $|x| \leq 1$ and $|\zeta| \leq\left|\zeta_{0}\right|$.

To obtain a criterion for the nonnegativity of $Q(x, \zeta)$ in $|x| \leq 1$ we have to determine the greatest possible $\zeta$ such that $|\zeta|<1$ and $Q(x, \zeta) \geq 0$ for $|x| \leq 1$.

An elementary computation shows that the function $Q(x, \zeta)$ has exactly one minimum in the interval $-1<x<+1$ which is located at $x=-\zeta$.

We consider next the equation $R(z) \equiv Q(-z, z)=0$ or, written in greater detail,

$\int_{-2}^{1}\left(1-t^{2}\right)^{n-1}\left(t^{2}-z^{2}\right) d t=P_{1}(-z)-z^{2} P_{0}(-z)=0$.

Clearly $R(0)>0$ while $R(1)=P_{1}(-1)-P_{0}(-1)<0$ so that the equation $(41)$ has at least one root in $(0,1)$. Moreover

$$
\frac{d R}{d z}=-2 z \int_{-z}^{1}\left(1-t^{2}\right)^{n-1} d t
$$

so that $d R / d z$ vanishes in the interval $0 \leq z<1$ only at the point $z=0$, therefore the equation (41) has exactly one root $z_{n}$ in the interval $(0,1)$.

Since $\min Q\left(x, z_{n}\right)=R\left(z_{n}\right)=0$ we see that $Q\left(x, z_{n}\right)$ $\geq 0$ in $|x| \leq 1$ and therefore $Q(x, \zeta) \geq 0$ in $|x| \leq 1$ if $\zeta \leq z_{n}$.

If however $\zeta>z_{n}$ then

$$
\begin{aligned}
Q\left(-z_{n}, \zeta\right) & =P_{1}\left(-z_{n}\right)-\zeta^{2} P_{0}\left(-z_{n}\right) \\
& =-\left(\zeta^{2}-z_{n}^{2}\right) P_{0}\left(-z_{n}\right)<0
\end{aligned}
$$


so that $Q(x, \zeta)$ assumes also negative values. This establishes the first part of lemma 3 . We still have to discuss the asymptotic behaviour of the solution of equation (41).

We derive first a useful inequality. By definition $z_{n}$ is a root of equation (41), therefore

$\int_{-1}^{+1}\left(1-t^{2}\right)^{n-1}\left(t^{2}-z_{n}^{2}\right) d t=\int_{-1}^{-z_{n}}\left(1-t^{2}\right)^{n-1}\left(t^{2}-z_{n}^{2}\right) d t>0$.

If we set

$$
w_{n}=\sqrt{n+\frac{1}{2}} z_{n}
$$

then we see that

$$
2 \int_{0}^{1}\left(1-t^{2}\right)^{n-1}\left(t^{2}-\frac{w_{n}^{2}}{n+\frac{1}{2}}\right) d t \geq 0 .
$$

or

$$
\int_{0}^{1}(1-t)^{n-1} t^{\frac{1}{2}} d t-\frac{w_{n}^{2}}{n+\frac{1}{2}} \int_{0}^{1}(1-t)^{n-1} t^{-\frac{1}{2}} d t \geq 0
$$

We see therefore that

$$
\frac{\Gamma(n) \Gamma\left(\frac{3}{2}\right)}{\Gamma\left(n+\frac{3}{2}\right)}-\frac{w_{n}^{2}}{n+\frac{1}{2}} \frac{\Gamma(n) \Gamma\left(\frac{1}{2}\right)}{\Gamma\left(n+\frac{1}{2}\right)} \geq 0
$$

so that

$$
w_{n}^{2} \leq \frac{1}{2} \text { or } z_{n}^{2} \leq \frac{1}{2 n+1} .
$$

In order to derive an asymptotic estimate for $w_{n}$ we rewrite (41) by dividing the interval of integration into two parts $(-z, 0)$ and $(0,1)$. The integrals over the second interval may be easily expressed in terms of gamma functions. A simple computation yields:

$$
\begin{aligned}
R(z) & =\frac{1}{2} \frac{\Gamma\left(\frac{1}{2}\right) \Gamma(n)}{\Gamma\left(n+\frac{1}{2}\right)}\left(\frac{1}{2 n+1}-z^{2}\right) \\
& -z^{3} \int_{0}^{1}\left(1-z^{2} t^{2}\right)^{n-1}\left(1-t^{2}\right) d t .
\end{aligned}
$$

If we write

$$
L(z)=\frac{2\left(n+\frac{1}{2}\right) \Gamma\left(n+\frac{1}{2}\right)}{\Gamma(n)} R(z)
$$

and

$$
I_{n}(z)=\int_{0}^{1}\left(1-z^{2} t^{2}\right)^{n-1}\left(1-t^{2}\right) d t
$$

we have

$L(z)=\frac{1}{2} \sqrt{\pi}\left[1-(2 n+1) z^{2}\right]-\frac{2 \Gamma\left(n+\frac{1}{2}\right)}{\Gamma(n)}\left(n+\frac{1}{2}\right) z^{3} I_{n}$.

From the asymptotic formula for $\log \Gamma(x)$ it is seen that

$$
\frac{\Gamma\left(n+\frac{1}{2}\right)}{\left(n+\frac{1}{2}\right)^{\frac{1}{2}} \Gamma(n)}=\exp \left[-\frac{3}{8 n}+o\left(n^{-1}\right)\right]=e^{\left(n^{-1}\right)}
$$

hence

$$
L(z)=\frac{\sqrt{\pi}}{2}\left[1-(2 n+1) z^{2}\right]-2 e^{\left(n^{-1}\right)}\left(n+\frac{1}{2}\right)^{\frac{3}{2}} z^{3} I_{n} .
$$

We next transform the integral $I_{n}$. Let $0<x<1$ then $(1-x) e^{x}<1$ and $(1-x)^{n-1}<e^{-(n-1) x}$, therefore

$$
0<1-\left[(1-x) e^{x}\right]^{n-1}<(n-1)\left[1-(1-x) e^{x}\right] .
$$

Since $e^{x}>1+x$ we have also $(1-x) e^{x}>1-x^{2}$ and $1-(1-x) e^{x}<x^{2}$ so that finally $0<1-(1-x)^{n-1} e^{(n-1) x}$ $<(n-1) x^{2}$ or $0<e^{-(n-1) x}-(1-x)^{n-1}<(n-1) x^{2}$. We replace here $x$ by $z^{2} t^{2}$ and see that $0<e^{-(n-1) z^{2} t^{2}-}$ $\left(1-z^{2} t^{2}\right)^{n-1}<(n-1) z^{4} t^{4}$ for $z^{2} t^{2}<1$, so that

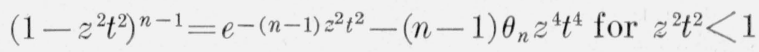

with $0<\theta_{n}<1$.

We substitute this into the expression (44) for $I_{n}$ and obtain

$$
I_{n}=\int_{0}^{1} e^{-(n-1) z^{2} t^{2}}\left(1-t^{2}\right) d t-\frac{2 \delta_{n}}{35}(n-1) z^{4}
$$

for $z^{2}<1$ with $0<\delta_{n}<1$.

We have

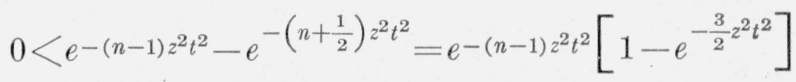

Since $0<1-e^{-\frac{3}{2} z^{2} t^{2}}<\frac{3}{2} z^{2} t^{2}$ we see that $\left(1-e^{-\frac{3}{2} z^{2} t^{2}}\right)$ $=\eta z^{2} t^{2}$ with $0<\eta<3 / 2$ and hence

$$
e^{-(n-1) z^{2} t^{2}}=e^{-\left(n+\frac{1}{2}\right) z^{2} t^{2}}+\eta e^{-(n-1) z^{2} t^{2} z^{2} t^{2}}
$$

therefore

$$
\begin{aligned}
\int_{0}^{1} e^{-(n-1) z^{2} t^{2}\left(1-t^{2}\right) d t} & =\int_{0}^{1} e^{-\left(n+\frac{1}{2}\right) z^{2} t^{2}}\left(1-t^{2}\right) d t \\
& +\frac{2 \lambda}{15} z^{2} e^{-(n-1) z^{2} \sigma}
\end{aligned}
$$

with $0<\sigma<1$ and $0<\lambda<\frac{3}{2}$. 
Substituting this into (46) we have

$$
\begin{aligned}
I_{n}= & \int_{0}^{1} e^{-\left(n+\frac{1}{2}\right) z^{2} t^{2}}\left(1-t^{2}\right) d t+ \\
& \frac{2 \lambda z^{2}}{15} e^{-(n-1) z^{2} \sigma}-\frac{2 \delta_{n}(n-1) z^{4}}{35}
\end{aligned}
$$

with $0<\delta_{n}<1,0<\sigma<1,0<\lambda<\frac{3}{2}$.

We introduce next a new variable $w_{n}=\sqrt{n+{ }_{2}^{1}} z$, using (45) and (47) we see that $w_{n}$ satisfies the equation

$$
\begin{gathered}
\sqrt{\pi}\left(\frac{1}{2}-w_{n}^{2}\right)-2 w_{n}^{3} e^{O\left(n^{-1}\right)} \int_{0}^{1} e^{-w_{n}^{2} t^{2}}\left(1-t^{2}\right) d t \\
-2 w_{n}^{3} e^{O(n-1)}\left[\frac{2 \lambda}{15} \frac{w_{n}^{2}}{n+\frac{1}{2}} e^{-\frac{n-1}{n+\frac{1}{2}} \sigma w_{n}^{2}}-\frac{2 \delta_{n} w_{n}^{4}}{35} \frac{(n-1)}{\left(n+\frac{1}{2}\right)^{2}}\right]=0
\end{gathered}
$$

For each value of $n$ this equation has a root $w_{n}$, by (43) we see that the set of these roots is bounded. We consider any accumulation point $\rho$ of this set and a subsequence $\left\{w_{n_{i}}\right\}$ of the sequence $\left\{w_{n}\right\}$ such that $\lim _{i \rightarrow \infty} w_{n_{i}}=\rho$. From (48) it is seen that $\rho$ satisfies the equation

$$
F(\rho)=\sqrt{\pi}\left(\frac{1}{2}-\rho^{2}\right)-2 \rho^{3} \int_{0}^{1} e^{-\rho^{2} t^{2}}\left(1-t^{2}\right) d t=0
$$

and by transforming the integral we obtain finally for $\rho$ the equation

$$
F(\rho)=\sqrt{\pi}\left(\frac{1}{2}-\rho^{2}\right)-\int_{0}^{\rho}\left(\rho^{2}-x^{2}\right) e^{-x^{2}} d x=0
$$

To complete the proof of lemma 3 we have only to show that the sequence $\left\{w_{n}\right\}$ converges. We dem- onstrate this by showing that $\left\{w_{n}\right\}$ has only one accumulation point, and this follows if we show that $F(\rho)=0$ has only one root in $\left(0, \sqrt{\frac{1}{2}}\right)$.

We see easily that $F(0)>0$ and $F\left(\sqrt{\frac{1}{2}}\right)<0$ so that there is at least one root in the interval.

From (50) we see that

$$
F^{\prime}(\rho)=-2 \rho\left[\sqrt{\pi}+2 \int_{0}^{\rho} e^{-x^{2}} d x\right]
$$

so that $F^{\prime}(\rho)<0$ for $\rho>0$. This shows that $F(\rho)$ has exactly one root.

The equation (41) has been evaluated for certain values of $n$, similarly the asymptotic solution $\rho$ has been found from $(50)$. This was done in part at the

\begin{tabular}{|c|c|c|}
\hline$n$ & $z_{n}$ & $w_{n}$ \\
\hline $\begin{array}{l}2 \\
3 \\
4 \\
5 \\
6\end{array}$ & $\begin{array}{l}38197 \\
32114 \\
.28244 \\
25505 \\
23434\end{array}$ & $\begin{array}{r}60394 \\
60079 \\
.59915 \\
.59814 \\
59745\end{array}$ \\
\hline $\begin{array}{r}7 \\
8 \\
9 \\
10 \\
15 \\
20\end{array}$ & $\begin{array}{l}21798 \\
20463 \\
19346 \\
18395 \\
15122 \\
13141\end{array}$ & $\begin{array}{r}59696 \\
.59659 \\
.59630 \\
.59606 \\
.59536 \\
.59500\end{array}$ \\
\hline \multicolumn{3}{|c|}{$\rho=0.5939157$} \\
\hline
\end{tabular}
Computation Laboratory of the National Bureau of Standards, Washington, D. C., and in part at the computation department of the Institute for Numerical Analysis in Los Angeles, Calif. The results are given in the following table, which shows also the values of $w_{n}$.

Washington, May 25, 1951 